\title{
CONNECTING LOCALLY COMPACT ABELIAN GROUPS
}

\author{
ED ENOCHS AND WALT GERLACH
}

\begin{abstract}
Those locally compact abelian groups having connected envelopes are characterized as those $G$ such that the dimension of $\operatorname{Hom}(G, R)$ over $R$ is finite (where $R$ is the field of real numbers).
\end{abstract}

In [1] J. Sonner initiated the study of the so-called weak universal and special problems. A somewhat broader notion is that of a very weak universal problem. Various examples of such are well known and the solutions are usually called envelopes in the case of right universal problems and covers in the left case. In [2] various covers and envelopes are considered, particularly in categories of modules. The following definition is sufficiently general for our purpose.

Definition. If $\mathcal{G}$ is a full subcategory of a category $\mathscr{F}$ and $A$ is an object of $\mathscr{F}$, a

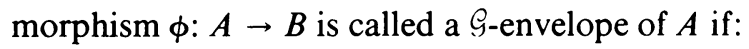

(1) $B$ is an object of $\mathcal{G}$, and for any morphism $A \rightarrow B^{\prime}$ with $B^{\prime} \in \mathcal{G}$ the diagram

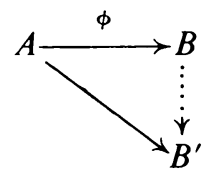

can be completed to a commutative diagram;

(2) the diagram

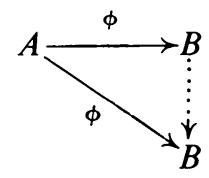

can be completed only by automorphisms of $B$. $\mathcal{G}$-covers are defined dually.

Note that covers and envelopes are unique up to isomorphism. A connected envelope of a locally compact abelian group $G$ is just the envelope we get by considering the full subcategory of connected groups in the category of all locally compact abelian groups. Similar terminology can easily be understood in other contexts by taking the obvious categories.

In [3] it was shown that every abelian group $G$ has a torsion-free cover, denoted $T(G) \rightarrow G$. By Pontrjagin duality this implies that every compact abelian group has a connected envelope. In fact if $K$ is compact abelian, then $K \rightarrow T\left(K^{*}\right)^{*}$ is the connected envelope (where $K^{*}$ denotes the Pontrjagin dual of $K$ ). In [4] Gerlach

Received by the editors May 13, 1982 and, in revised form, January 7, 1983.

1980 Mathematics Subject Classification. Primary 22B05. 
showed that every compactly generated group has a connected envelope in the category of all locally compact abelian groups, but left open the question for an arbitrary group.

We now have

THEOREM. A locally compact abelian group $G$ has a connected envelope if and only if the dimension of $\operatorname{Hom}(G, R)$ over $R$ is finite.

Proof. Necessity. Suppose $G$ has a connected envelope. Since every connected locally compact abelian group has the form $R^{k} \times C$, where $C$ is compact and connected, suppose $G \rightarrow R^{k} \times C$ is an envelope. If $\phi_{1}, \phi_{2}, \ldots, \phi_{m} \in \operatorname{Hom}(G, R)$ are linearly independent over $R$ then $\phi=\left(\phi_{1}, \ldots, \phi_{n}\right): G \rightarrow R^{n}$ is such that $\phi(G)$ contains a base of $R^{n}$. If then we complete

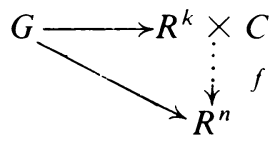

we see that $f(C)=0$ and so $f\left(R^{k}\right) \subset R^{n}$ contains a base of $R^{n}$. Consequently $f\left(R^{k}\right)=R^{n}$ and so $k \geqslant n$. Hence $\operatorname{dim} \operatorname{Hom}(G, R) \leqslant k$.

Now assume $\operatorname{Hom}(G, R)<\infty$. We construct a connected envelope of $G$. The precedure is to consider a compact connected envelope of $G$ and to modify this to obtain the desired envelope. The following gives a standard construction of the Bohr compactification of $G$, denoted $\hat{G}$, considered as a solution of the obvious universal problem.

LEMma 1. $\hat{G}=\left(\left(G^{*}\right)_{0}\right)^{*}$ (where $\left(G^{*}\right)_{0}$ denotes $G^{*}$ with the discrete topology).

Proof. With the obvious map $G \rightarrow \hat{G}$ we need to show we can complete

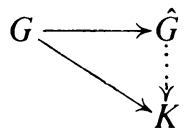

uniquely where $K$ is compact abelian. By Pontrjagin duality this is equivalent to completing

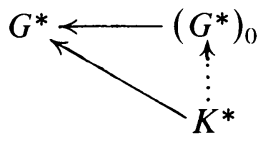

But this is trivial since $\left(G^{*}\right)_{0} \rightarrow G^{*}$ is the identity map.

We note that from the description of connected groups it easily follows that the connected envelope, say $K \rightarrow C$, of a compact group $K$ in the category of compact abelian groups is in fact a connected envelope in the category of locally compact abelian groups. This is because $\operatorname{Hom}\left(C, R^{k}\right)=0$.

Hence we have

LEMMA 2. Every locally compact abelian group $G$ has a compact connected envelope.

Proof. By chasing diagrams it is easy to see that the connected envelope of the Bohr compactification of $G$ is such an envelope. 
We now proceed to the construction of the connected envelope of $G$.

From the above we see that the compact connected envelope is

$$
T\left(\hat{G}^{*}\right)^{*}=T\left(\left(G^{*}\right)_{0}^{* *}\right)^{*}=T\left(\left(G^{*}\right)_{0}\right)^{*} .
$$

But $T\left(\left(G^{*}\right)_{0}\right) \rightarrow\left(G^{*}\right)_{0}$ is a torsion-free cover, so the natural map $\operatorname{Hom}(G, R)_{0} \rightarrow$ $\operatorname{Hom}(G, R / Z)_{0}=\left(G^{*}\right)_{0}$ can be factored through $T\left(\left(G^{*}\right)_{0}\right)$ since $\operatorname{Hom}(G, R)_{0}$ is torsion free. The kernel of the map $\operatorname{Hom}(G, R)_{0} \rightarrow T\left(\left(G^{*}\right)_{0}\right)$ is contained in $\operatorname{Hom}(G, Z)$, which is clearly a discrete subgroup of the vector group $\operatorname{Hom}(G, R)$. Since $T\left(\left(G^{*}\right)_{0}\right)$ is torsion free the kernel of $\operatorname{Hom}(G, R)_{0} \rightarrow T\left(\left(G^{*}\right)_{0}\right)$ must be 0 . $\operatorname{Hom}(G, R)_{0}$ is divisible so it is a direct summand of $T\left(\left(G^{*}\right)_{0}\right)$, say

$$
T\left(\left(G^{*}\right)_{0}\right)=\operatorname{Hom}(G, R)_{0} \oplus L \text {. }
$$

Then $T\left(\left(G^{*}\right)_{0}\right)^{*}=\left(\operatorname{Hom}(G, R)_{0}\right)^{*} \oplus L^{*}$. Note that $\left(\operatorname{Hom}(G, R)_{0}\right)^{*}$ is the Bohr compactification of the vector group $\operatorname{Hom}(\operatorname{Hom}(G, R), R)$ since

$$
\operatorname{Hom}(\operatorname{Hom}(G, R), R)^{*}=\operatorname{Hom}(G, R) \text {. }
$$

We claim that the obvious map $G \rightarrow \operatorname{Hom}(\operatorname{Hom}(G, R), R) \oplus L^{*}$ is the desired envelope. To argue that

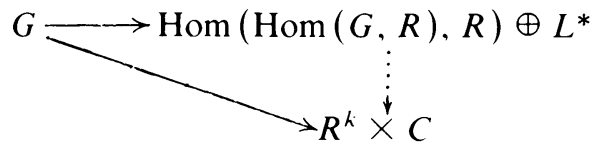

can always be completed (where $C$ is compact and connected) we consider the case $R^{k}$ and $C$ separately. In fact

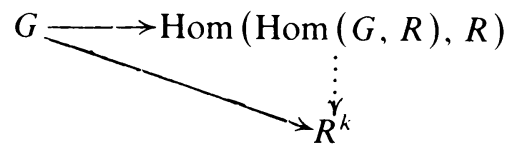

can be completed uniquely since $\operatorname{Hom}\left(\operatorname{Hom}(\operatorname{Hom}(G, R), R) R^{k}\right) \cong \operatorname{Hom}\left(G, R^{k}\right)$ (naturally). For $C$ we consider the extended diagram

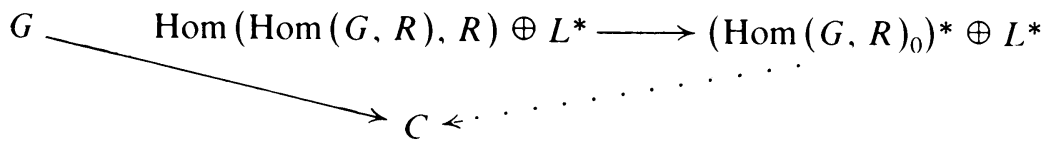

Since the horizontal composition is a compact connected envelope, we can complete the diagram. To finish the proof we argue that if $\phi$ completes

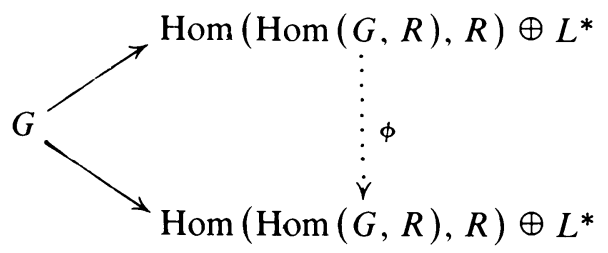

then $\phi$ is an automorphism. Using matrix notation let

$$
\phi=\left(\begin{array}{ll}
\phi_{11} & \phi_{12} \\
\phi_{21} & \phi_{22}
\end{array}\right) \text {. }
$$


Then $\phi_{12}: L^{*} \rightarrow \operatorname{Hom}(\operatorname{Hom}(G, R), R)$ is 0 since $L^{*}$ is compact. Furthermore $\phi_{11}$ completes

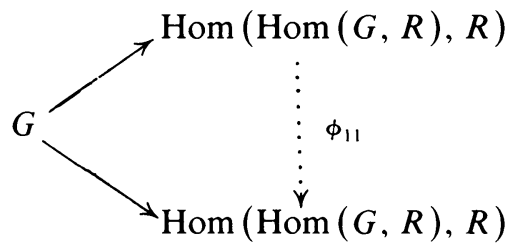

and so $\phi_{11}=$ id. Thus

$$
\phi=\left(\begin{array}{cc}
\text { id } & 0 \\
\phi_{21} & \phi_{22}
\end{array}\right)
$$

and we only have to show that $\phi_{22}$ is an automorphism of $L^{*}$. For this consider the extended commutative diagram

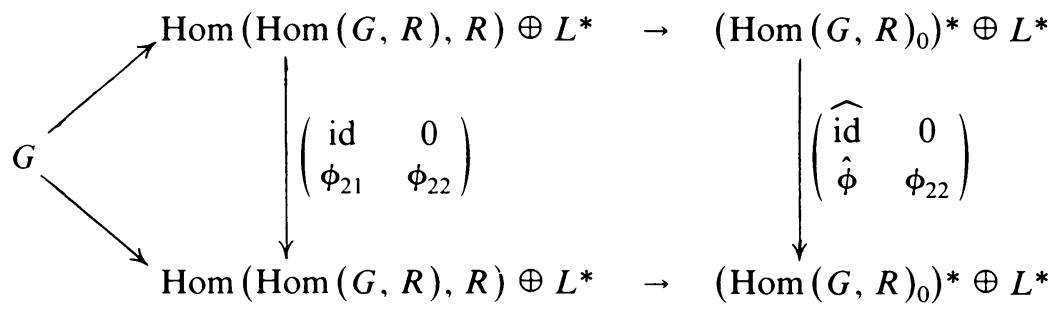

where we get $\hat{\phi}_{21}$ and $\widehat{\mathrm{id}}$ by regarding $\left(\operatorname{Hom}(G, R)_{0}\right)^{*}$ as the Bohr compacitifaction of $\operatorname{Hom}(\operatorname{Hom}(G, R), R)$. Then since $G \rightarrow\left(\operatorname{Hom}(G, R)_{0}\right)^{*} \oplus L^{*}$ is a compact connected envelope,

$$
\left(\begin{array}{cc}
\widehat{\mathrm{id}} & 0 \\
\hat{\phi}_{21} & \phi_{22}
\end{array}\right)
$$

is an automorphism, and consequently $\phi_{22}$ is an automorphism of $L^{*}$. This completes the proof.

\section{REFERENCES}

1. J. Sonner, Universal and special problems. Math. Z. 82 (1963), 200-211.

2. E. Enochs, Injective and flat covers, envelopes and resolvants, Israel J. Math 39 (1981), 189-209.

3. __ Torsion free covering modules, Proc. Amer. Math. Soc. 14 (1963), 884-889.

4. W. Gerlach, Connecting locally compact abelian groups, Ph. D. thesis, Univ. of Kentucky, 1980.

Department of Mathematics, University of Kentucky, Lexington, Kentucky 40506 (Current address of Ed Enochs)

Department of Mathematics, Centre College, Danville, Kentucky 40422

Current address (Walt Gerlach): Department of Mathematics, Columbus College, Columbus, Georgia 31993 